\title{
Convergence of Multiple Stimuli to a Single Gate in TREK1 and TRAAK Potassium Channels
}

\author{
Frank S Choveau ${ }^{\dagger}$, Ismail Ben Soussia ${ }^{\dagger}$, Delphine Bichet, Chatelain C. Franck, \\ Sylvain Feliciangeli and Florian Lesage*
}

Université Côte D'Azur, INSERM, CNRS, Institut de Pharmacologie Moléculaire et Cellulaire, LabEx ICST, Valbonne, France

\section{OPEN ACCESS}

Edited by:

Bernard Attali,

Tel Aviv University, Israel

Reviewed by:

Rían Manville,

University of Brighton,

United Kingdom

Alistair Mathie,

University of Kent, United Kingdom

*Correspondence:

Florian Lesage

lesage@ipmc.cnrs.fr

${ }^{t}$ These authors have contributed equally to this work and share first

authorship.

Specialty section:

This article was submitted to Pharmacology of lon Channels and

Channelopathies,

a section of the journal

Frontiers in Pharmacology

Received: 09 August 2021 Accepted: 15 September 2021 Published: 30 September 2021

Citation:

Choveau FS, Ben Soussia I, Bichet D, Franck CC, Feliciangeli S and Lesage F (2021) Convergence of Multiple Stimuli to a Single Gate in TREK1 and TRAAK

Potassium Channels.

Front. Pharmacol. 12:755826. doi: 10.3389/fphar.2021.755826
Inhibitory potassium channels of the TREK1/TRAAK family are integrators of multiple stimuli, including temperature, membrane stretch, polyunsaturated fatty acids and $\mathrm{pH}$. How these signals affect the gating of these channels is the subject of intense research. We have previously identified a cytoplasmic domain, pCt, which plays a major role in controlling channel activity. Here, we use pharmacology to show that the effects of pCt, arachidonic acid, and extracellular pH converge to the same gate within the channel. Using a statedependent inhibitor, fluoxetine, as well as natural and synthetic openers, we provide further evidence that the "up" and "down" conformations identified by crystallography do not correspond to open and closed states of these channels.

Keywords: two-pore domain potassium channels, pore gating, openers, allostery, extracellular pH

\section{INTRODUCTION}

TWIK-related $\mathrm{K}^{+}$channels TREK1, TREK2 and TRAAK are two-pore-domain potassium $\left(\mathrm{K}_{2 \mathrm{P}}\right)$ channels (Enyedi and Czirjak, 2010; Feliciangeli et al., 2015). Active as homo or heterodimers (Blin et al., 2016; Lengyel et al., 2016; Levitz et al., 2016), they play important roles in the nervous system, regulating cognitive function, pain perception, neuroprotection as well as anesthesia (Heurteaux et al., 2004; Noel et al., 2011; Wang et al., 2020). They produce inhibitory background currents that are sensitive to a wide range of physical and biological stimuli including mechanical forces, temperature, $\mathrm{pH}$ and lipids (Maingret et al., 1999; Maingret et al., 2000a; Maingret et al., 2000b; Honore et al., 2002; Sandoz et al., 2009). They are sensitive to antidepressants and volatile anesthetics (Patel et al., 1999; Heurteaux et al., 2006). These stimuli converge on a gate that involves the selectivity filter (SF) of these channels (Bagriantsev et al., 2011). The use of quaternary ammonium (QA) ions demonstrated that unlike most $\mathrm{K}^{+}$channels, in a closed state, TREK1 has no inner gate preventing the binding of QA ions deep within the pore just below SF (Piechotta et al., 2011). Intracellular acidification (Sandoz et al., 2009; Piechotta et al., 2011), mechanical forces (Bagriantsev et al., 2011) or binding of the cytoplasmic protein AKAP150 (Sandoz et al., 2006) all act indirectly on the SF gate through the fourth membrane-spanning segment (M4) and the cytoplasmic C-ter of the channel (Bagriantsev et al., 2012). TREK1, TREK2 and TRAAK are also sensitive to the extracellular pH (Sandoz et al., 2009). A single extracellular residue, H126 in TREK1 and H151 in TREK2, is involved in proton sensing. TREK1 and TRAAK are inhibited by acidification whereas TREK2 is activated. This differential effect of acidification involves charged residues close to M4 (Sandoz et al., 2009). Structural modeling and site-directed mutagenesis suggested that attraction or repulsion between the protonated side chain of histidine and these closely located negatively or positively charged residues control the gating of these channels.

The recent crystallographic 3-D structures of these channels have shown that TREK/TRAAK channels can adopt two main conformational states which show differences in the positions of the 
membrane-spanning segments M2 and M4, and described as the "up" and "down" conformations (Brohawn et al., 2012; Lolicato et al., 2014). Although initially attributed to open and closed states, both conformations are in fact associated with channel activity. More recently, we showed that a cytoplasmic domain, immediately following M4 (the proximal C-ter domain, named pCt), exerts opposite effects in TREK1 and TRAAK (Soussia et al., 2018). In basal conditions, pCt favors activity in TREK1 whereas it impairs activity in TRAAK. Using the conformation-dependent binding of the antidepressant fluoxetine, we gathered data suggesting that TREK1 and TRAAK conformations at rest are different, "down" for TREK1 and "up" for TRAAK, and under the influence of pCt. The differential regulation of TREK1 and TRAAK is related to a phosphatidylinositol-4, 5-bisphosphate $\left(\mathrm{PIP}_{2}\right)$-binding site (R329, R330, R331) present within the pCt of TREK1 and absent in TRAAK. The binding of $\mathrm{PIP}_{2}$ to this site results in a stabilization of the conductive conformation of TREK1 (Soussia et al., 2018). Uncoupling pCt and M4 by introducing glycine residues at the junction of these domains prevents the activating property of TREK1 pCt and the inhibitory effect of TRAAK pCt, suggesting a physical coupling between $\mathrm{pCt}$ and the SF gate of the channel.

Despite all these data related to the 3D-structures and regulations of these channels, the nature of the conformational changes that control the activity of these channels remains an open question. Here, by using domain swapping and the conformation-dependent binding of fluoxetine, we evaluated how pCt affects the sensitivity of TREK1 and TRAAK to external $\mathrm{pH}$ and openers including ML67, BL1249, and halothane, a volatile anesthetic.

\section{MATERIAL AND METHODS}

Molecular biology - Human TREK1 (KCNK2, GenBank accession number AAH69462.1) and TRAAK (KCNK4, NCBI Reference Sequence: NP_201, 567.1) were cloned into pIRES2eGFP vector (Clontech). All the chimeras were obtained by overlapping PCR and inserted into the same vector. Substitution of I292G293D294 in TREK1 and I253G254N255 in TRAAK by a repeat of glycines was performed by PCR using Pfu Turbo DNA polymerase (Agilent). All the constructs were verified by DNA sequencing. The exchange of $\mathrm{pCt}$ between TREK1 and TRAAK to generate TRAAKpCt ${ }_{\text {TREK1 }}$ and TRAAKpCt $\mathrm{TREK}_{\text {TRE }}$ has been described elsewhere (Soussia et al., 2018).

Cell culture and transfection - HEK cells were grown in 100$\mathrm{mm}$ tissue-culture dishes (Falcon, Franklin Lakes, NJ) in Dulbecco's modified Eagle's medium (Gibco, Life Technologies, Saint Aubin, France) supplemented with 10\% fetal calf serum (Hyclone, Thermo Fisher Scientific GMBH, Ulm, Germany) and 1\% penicillin-streptomycin (Gibco, Life Technologies, Saint Aubin, France) in a humidified incubator at $37 \mathrm{C}(5 \% \mathrm{CO} 2)$. For expression and electrophysiology of WT and mutant channels, $0.8 \mu \mathrm{g}$ of plasmid was transfected using JET PEI (Polyplus transfection) according to the manufacturer's instructions. Cells were plated onto $35 \mathrm{~mm}$ dishes $24 \mathrm{~h}$ before transfection, and experiments were performed over the following 1-2 days.

Electrophysiology - Pipettes were pulled from haematocritcapillaries (Hirschmann Laborgeraete, Germany) using a vertical puller (PC-10, Narishige International, London, United Kingdom), and had resistances of 2-4 $\mathrm{M} \Omega$ when filled with internal solution and measured in standard bath solution. Whole cell membrane currents were measured and filtered at $3 \mathrm{kHz}$ by a RK 400 patch clamp amplifier (Bio-Logic Science Instruments), and digitized at $10 \mathrm{kHz}$ using a 12-bit analog-todigital converter Digidata-1322 (Axon Instrument, Sunnyvale, CA, United States of America). Recordings were done using Clampex 8.2 software (Axon Instrument). The external solution used to record TREK1 and TRAAK currents in HEK cells contained (in $\mathrm{mM}$ ): $140 \mathrm{NaCl}, 10 \mathrm{TE} \mathrm{A}-\mathrm{Cl}, 5 \mathrm{KCl}$, three $\mathrm{MgCl}_{2}$, one $\mathrm{CaCl}_{2}, 10$ HEPES, pH 7.4 with $\mathrm{NaOH}$. The pipette solution contained (in $\mathrm{mM}$ ): $155 \mathrm{KCl}$, three $\mathrm{MgCl}_{2}$, five EGTA, 10 HEPES, pH 7.2 with $\mathrm{KOH}$. Cells were placed in $35 \mathrm{~mm}$ dishes through which solution flowed at 1-2 $\mathrm{ml} / \mathrm{min}$. Inflow to the dish was by gravity from several reservoirs, selectable by activation of solenoid valves (Warner Scientific). Bath solution exchange was essentially complete by $<30 \mathrm{~s}$. Experiments were performed at room temperature. Cell populations were compared using Kruskall Wallis, Mann-Whitney test or Two-way ANOVA. The data are given as the mean \pm SEM.

\section{RESULTS}

\section{Openers and pCt Have Allosteric Effects on the Channel Activity of TREK1 and TRAAK}

To study the impact of pCt on the activating effects of openers, we expressed chimeric channels in which pCt is swapped between TREK1 (residues W295 to A343) and TRAAK (residues W256 to P302), to give TREK1pCt $t_{\text {TRAAK }}$ and TRAAKpCt $t_{\text {TREK1 }}$ (Figure 1). To physically uncouple the M4 and pCt domains in these channels, residues 292 to 294 in TREK1 were replaced by three glycines to produce TREK1-3G and residues 253 to 255 in TRAAK to produce TRAAK-3G (Figure 1).

We first studied the sensitivity of TREK1 and TRAAK to the opener ML67 (Figure 2) (Bagriantsev et al., 2013). ML67 stimulates by nearly 5 -fold TREK1 $\left(\mathrm{I}_{\mathrm{drug}} / \mathrm{I}_{\text {control }}=4.76 \pm 0.43\right.$, Figures $\left.2, \mathbf{5 A}\right)$. Under the same conditions, ML67 is even more efficient on TRAAK with a 12 -fold increase $\left(\mathrm{I}_{\mathrm{drug}} / \mathrm{I}_{\text {control }}=12.38 \pm 1.26\right)$. Swapping $\mathrm{pCt}$ between TREK1 and TRAAK reverses their sensitivity to ML67: TREK1pCt $\mathrm{TRAAK}_{\text {TRA }}$ becomes more sensitive than TREK1 ( $\mathrm{I}_{\text {drug }} / \mathrm{I}_{\text {control }}$ $=13.97 \pm 1.73$ versus $\left.\mathrm{I}_{\text {drug }} / \mathrm{I}_{\text {control }}=4.76 \pm 0.43\right)$, whereas TRAAKpCt $_{\text {TREK1 }}$ becomes less sensitive than TRAAK $\left(\mathrm{I}_{\text {drug }} /\right.$ $\mathrm{I}_{\text {control }}=3.80 \pm 0.44$ versus $\mathrm{I}_{\text {drug }} / \mathrm{I}_{\text {control }}=12.38 \pm 1.26$, Figures 2, 5A). Uncoupling pCt from the SF gate, by introducing glycine residues between $\mathrm{pCt}$ and $\mathrm{M} 4$, also affects the sensitivity of TRAAK to ML67. TRAAK-3G is significantly less sensitive to ML67 than TRAAK $\left(\mathrm{I}_{\text {drug }} / \mathrm{I}_{\text {control }}=6.05 \pm 0.64\right.$ versus $\mathrm{I}_{\text {drug }} / \mathrm{I}_{\text {control }}$ $=12.38 \pm 1.26)$. The $3 \mathrm{G}$-mutation has no effect on TREK1 sensitivity to ML67 (Figures 2, 5A). 


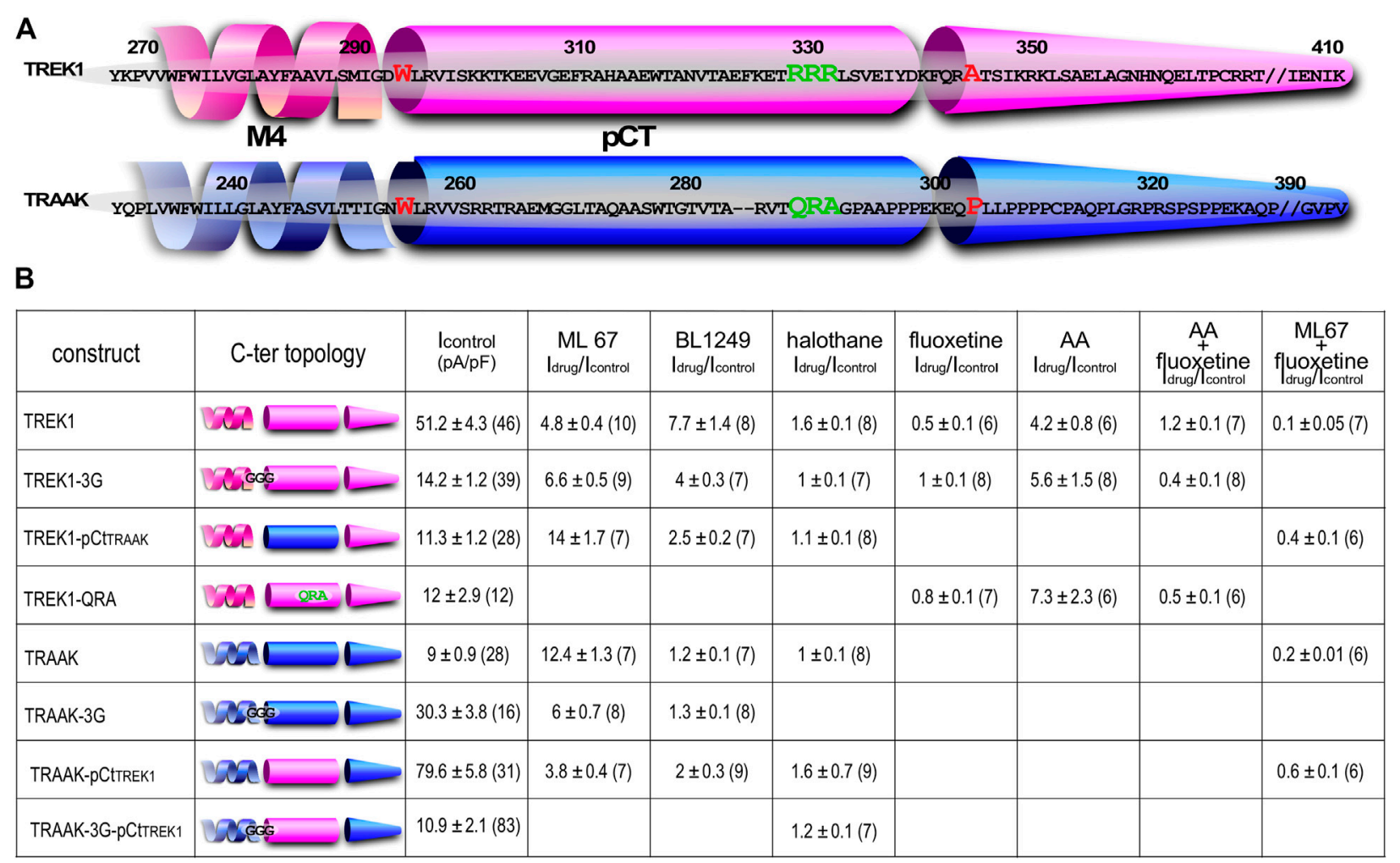

FIGURE 1 | Sequence alignment and Idrug/lcontrol ratios. (A) Alignment of human TREK1 and TRAAK (M4: membrane-spanning segment 4, pCt: proximal C-ter domain). The residues (IGD for TREK1 and IGN of TRAAK) substituted by three glycines in TREK1-3G and TRAAK-3G mutants are shown. The residues flanking TREK1 pCt (W295 and A343) and TRAAK pCt (W256 and P302) are in red. The residues forming a PIP 2 binding site in TREK1 (RRR), and the analogous residues (QRA) in TRAAK, are in green. This triplet R229-R230-R231 is substituted by QRA in TREK1-QRA. (B) I Irug $I_{\text {control }}$ ratios of the corresponding channels. I drug and I $I_{\text {control }}$ are the currents measured at $0 \mathrm{mV}(\mathrm{pA} / \mathrm{pF})$ in the presence and absence of drug as specified.

Next, we studied the sensitivity of TREK1 and TRAAK to the opener BL1249 (Tertyshnikova et al., 2005). As previously shown, TREK1 is more sensitive to BL1249 than TRAAK $\left(\mathrm{I}_{\text {drug }} / \mathrm{I}_{\text {control }}=\right.$ $7.7 \pm 1.4 \mathrm{vs} \mathrm{I}_{\text {drug }} / \mathrm{I}_{\text {control }}=1.16 \pm 0.03$, Figures 3, 4B) (Pope et al., 2018). The sensitivity of TRAAKpCtTREK1 to BL1249 is significantly higher than that of TRAAK $\left(\mathrm{I}_{\text {drug }} / \mathrm{I}_{\text {control }}=2.04 \pm\right.$ 0.31 vs $\left.\mathrm{I}_{\text {drug }} / \mathrm{I}_{\text {control }}=1.16 \pm 0.03\right)$, and TREK1pCtTRAAK has a lower sensitivity to BL1249 than TREK1 $\left(\mathrm{I}_{\text {drug }} / \mathrm{I}_{\text {control }}=2.49 \pm\right.$ 0.32 vs $\mathrm{I}_{\text {drug }} / \mathrm{I}_{\text {control }}=7.7 \pm 1.4$, Figures 3, 4B). Uncoupling $\mathrm{pCt} /$ M4 using the 3G-mutation decreases the sensitivity of TREK1 to BL1249 ( $\mathrm{I}_{\text {drug }} / \mathrm{I}_{\text {control }}=3.95 \pm 0.32$ vs $\left.\mathrm{I}_{\text {drug }} / \mathrm{I}_{\text {control }}=7.7 \pm 1.4\right)$ as shown previously by Pope et al. (2018), but had no effect on that of TRAAK $\left(\mathrm{I}_{\text {drug }} / \mathrm{I}_{\text {control }}=1.32 \pm 0.04 \mathrm{vs} \mathrm{I}_{\text {drug }} / \mathrm{I}_{\text {control }} 1.16 \pm 0.03\right.$, Figures 3, 4B).

We also tested the sensitivity of these channels to halothane, a volatile anesthetic. As previously reported, halothane activates TREK1 but not TRAAK (Patel et al., 1999) (Figure 4). TREK1pCt TRAAK $_{\text {has a lower sensitivity }}$ to halothane $\left(\mathrm{I}_{\mathrm{drug}} / \mathrm{I}_{\text {control }}=1.08 \pm 0.07\right)$ than TREK1 (Idrug/ Icontrol $=1.59 \pm 0.08)$, whereas TRAAKpCt $\mathrm{TREK}_{\mathrm{TR} 1}$ exhibits sensitivity to halothane $\left(\mathrm{I}_{\text {drug }} / \mathrm{I}_{\text {control }}=1.59 \pm 0.07\right.$, Figures 4, 5C) that is not present in TRAAK $\left(\mathrm{I}_{\text {drug }} / \mathrm{I}_{\text {control }}=1.03 \pm 0.06\right)$. We also evaluated the effect of the 3G-mutation on the sensitivity of TREK1 and TRAAK to halothane. TREK1-3G is no longer sensitive to the drug $\left(\mathrm{I}_{\mathrm{drug}} / \mathrm{I}_{\text {control }}=1.03 \pm 0.07\right.$, Figures 4, 5C). Furthermore, the $3 \mathrm{G}$-mutation also rendered TRAAKpCt $_{\text {TREK } 1}$ insensitive to halothane as TRAAK ( $\mathrm{I}_{\mathrm{drug}} /$ $\mathrm{I}_{\text {contr }} \mathrm{Ol}=1.16 \pm 0.05$, Figures $\left.4,5 \mathrm{C}\right)$, showing that $\mathrm{pCt} / \mathrm{M} 4$ coupling is necessary for halothane action on TREK1 and TRAAKPCt $\mathrm{T}_{\text {TREK1 } 1}$.

These results show that pCt alters the sensitivity of TREK1 and TRAAK channels to ML67, BL1249 and halothane. The alteration is unique to each opener. Thus, ML67 is more active on TRAAK than on TREK1. The pCt of TRAAK favors activation by ML67 because its introduction into TREK1 increases its activity and its uncoupling to M4 decreases TRAAK sensitivity. Unlike TRAAK pCt, TREK1 pCt has no effect, and its uncoupling to M4 in TREK1-3G does not affect the effect of ML67. Unlike ML67, BL1249 is more active on TREK1 than on TRAAK. TREK1 pCt favors activation by BL1249. Its introduction into TRAAK makes this channel more sensitive to BL1249 whereas its uncoupling to M4 decreases the sensitivity of TREK1 to BL1249. Finally, halothane activates TREK1 but not TRAAK. TREK1 pCt is required for activation by halothane and makes TRAAKpCt $_{\text {TREK1 }}$ sensitive to this drug. As expected, its uncoupling to $\mathrm{M} 4$ in TRAAK-3G-pCt $\mathrm{TREK1}_{\text {TRE }}$ suppresses this 


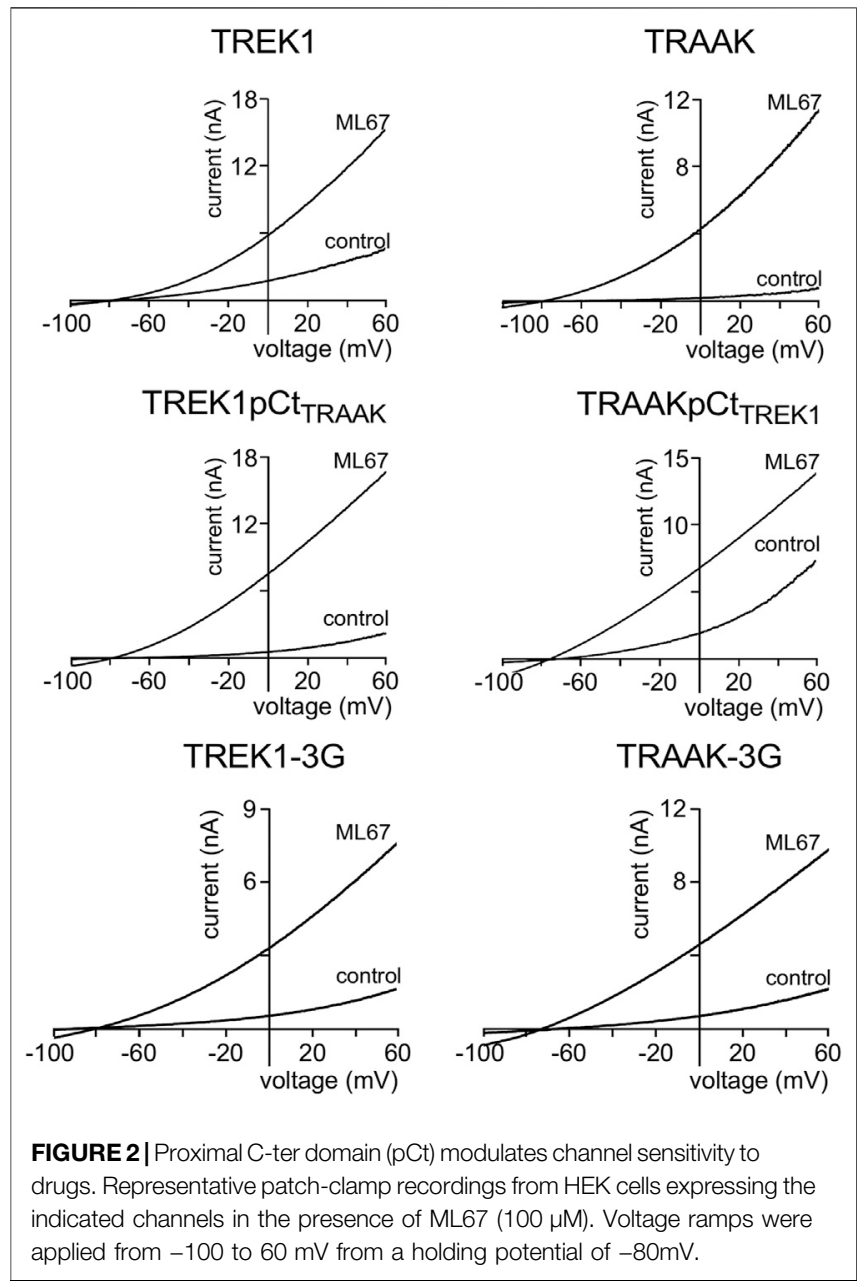

effect. The recent crystal structure of TREK2 interacting with BL1249 showed that this opener binds below the SF to the "fenestration site" at the interface of the M2/M3/M4 domains, likely stabilizing the SF. Like BL1249, ML67 has also been suggested to bind to the "fenestration site" (Schewe et al., 2019), suggesting that both openers act through a similar mechanism. Halothane was suggested to bind to the cytoplasmic C-ter of TREK1 because deletion of the last 48 residues of the C-ter completely abolished channel activation. However, insertion of these same residues into the C-ter of TRAAK did not confer halothane sensitivity (Patel et al., 1999), suggesting that the C-ter influences binding of volatile anaesthetics, but may not be the primary binding site. In full agreement with this, a recent study showed that volatile anaesthetics bind to a pocket formed by the G182 residue located in M2 and the M3-M4 domains, stabilizing the SF gate of the channel (Wague et al., 2020). Together, these data suggest that the effect of these three openers all converges on the same gate that is regulated by pCt. Beside this "distant" allosteric effect of pCt, we cannot exclude that this domain contains residues that may be also directly involved in the effect of the openers, contributing to their binding to the channels or modulating their effect on $\mathrm{K}^{+}$permeation.

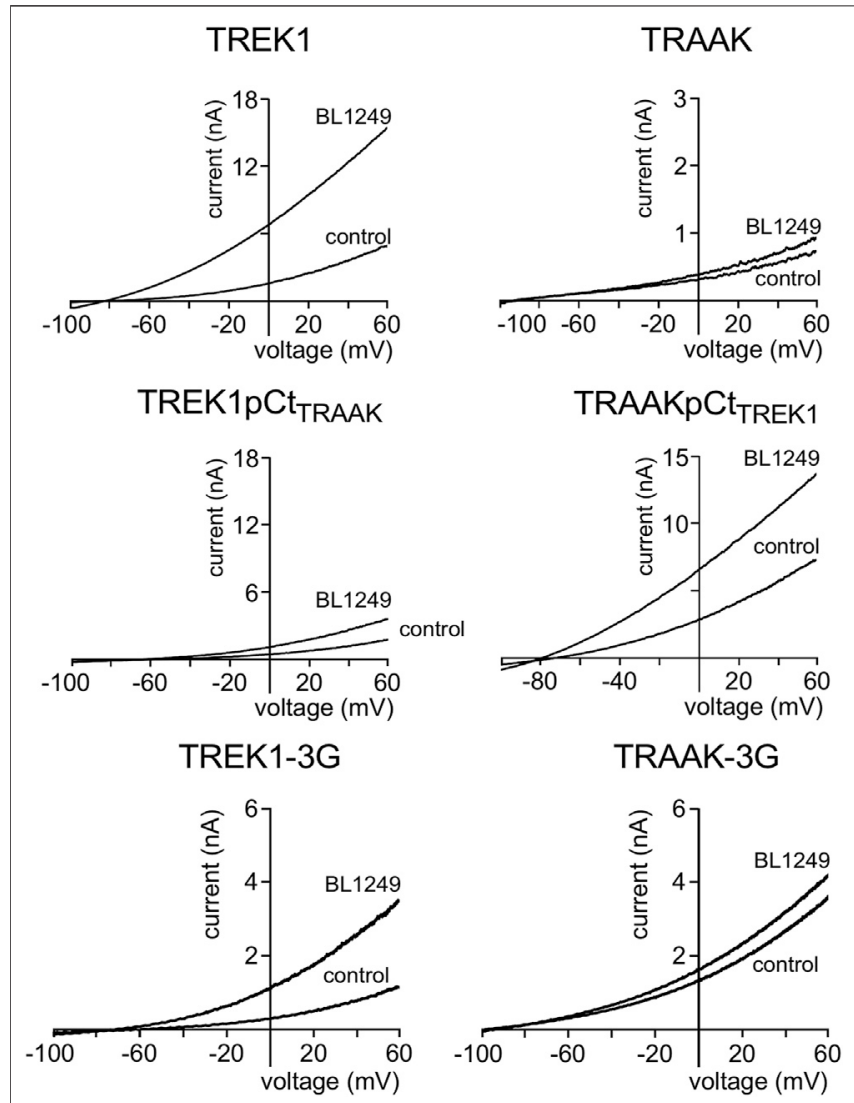

FIGURE 3|Proximal C-ter domain (pCt) modulates channel sensitivity to drugs. Representative patch-clamp recordings from HEK cells expressing the indicated channels in the presence of BL1249 (10 $\mu \mathrm{M})$. Voltage ramps were applied from -100 to $60 \mathrm{mV}$ from a holding potential of $-80 \mathrm{mV}$.

\section{Openers and pCt Have Allosteric Effects on the "up" and "Down" Conformations of TREK1 and TRAAK}

In a previous work (Soussia et al., 2018), we showed that pCt modulates the "up" and "down" conformations of TREK1 and TRAAK channels. Since different openers appear to control the same gate in these channels, we next addressed the possibility that openers affect both the "up" and "down" conformations. Using fluoxetine state-dependent binding, we first probed the conformation of TREK1. Under basal condition, TREK1 is in the fluoxetine-sensitive "down" conformation (Figures 6A,B). Unlike TREK1, TREK1-3G behaves like TRAAK that is in a fluoxetine-resistant "up" conformation. Fluoxetine has no effect on the TREK1-3G $\left(\mathrm{I}_{\text {fluoxetine }} / \mathrm{I}_{\text {control }}=0.97 \pm 0.05\right)$. However, upon application of arachidonic acid (AA), TREK1-3G becomes sensitive to fluoxetine $\left(\mathrm{I}_{\text {fluoxetine }} / \mathrm{I}_{\text {control }}=0.42 \pm 0.11\right.$, Figures 6C,D), suggesting that this mutated channel enters the "down" conformation once activated by AA. These results extend our previous ones (Soussia et al., 2018) and show that pCt contributes to the modulation of the conformational state through its coupling to M4. The QRA mutation in the pCt of TREK1 


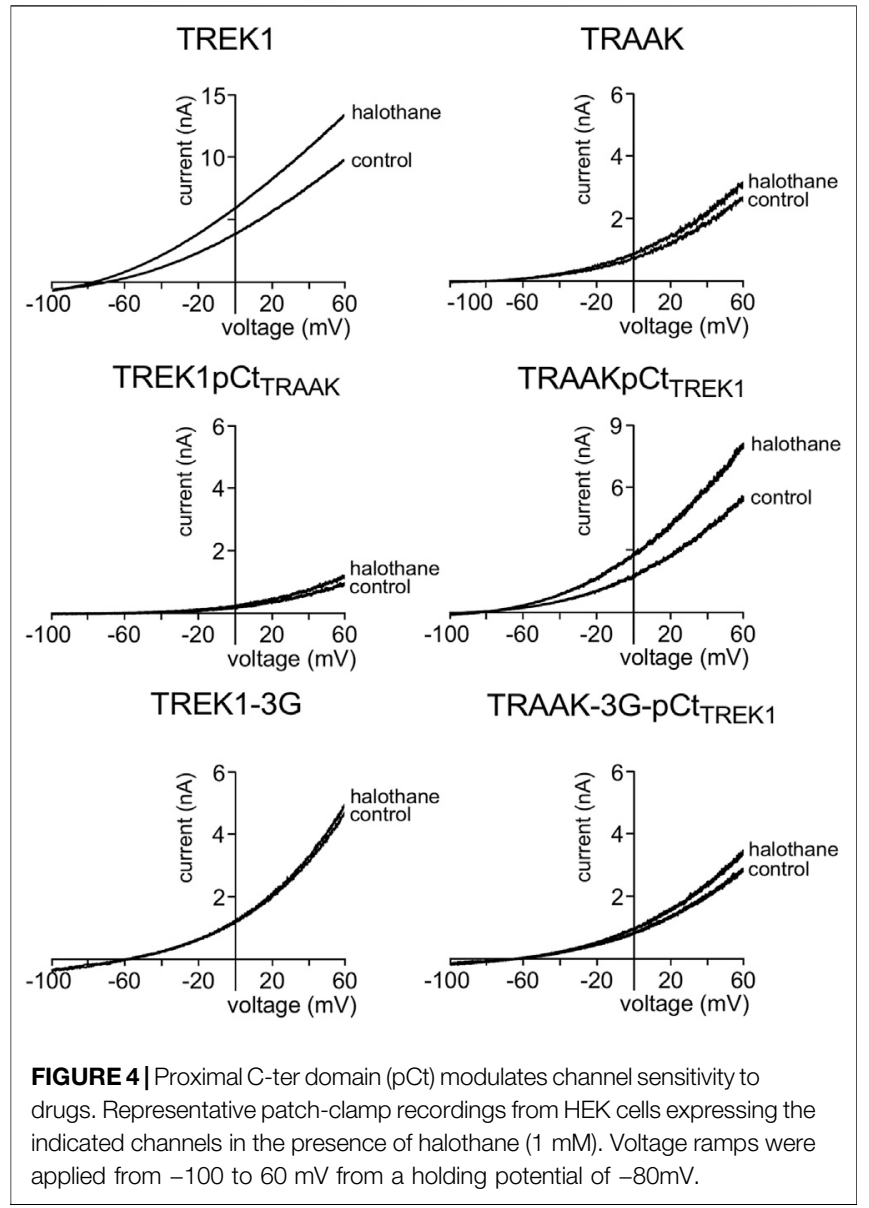

disrupts a potential site of interaction with $\mathrm{PIP}_{2}$ (R329, R330, R331) destabilizing the channel conductive state. TREK1QRA is less active than TREK1. We tested the sensitivity of TREK1-QRA in basal conditions and once activated by AA. Like TRAAK, TREK1-QRA is resistant to fluoxetine under basal conditions, suggesting that this mutated channel is in the "up" state (Figures 6E,F). Upon application of AA, TREK1-QRA reaches a fluoxetine-sensitive "down" state $\left(\mathrm{I}_{\text {fluoxetine }} / \mathrm{I}_{\text {control }}=0.52 \pm 0.04\right.$, Figures $\left.6 \mathrm{E}, \mathrm{F}\right)$ confirming that the $\mathrm{PIP}_{2}$-binding site in $\mathrm{pCt}$ modulates the conformational state of TREK1.

We then asked whether other openers could also induce changes in channel conformation in a pCt-dependent mechanism. Under basal condition, TREK1 is in the fluoxetine-sensitive "down" conformation (Figures 6A,B) whereas TRAAK is in the fluoxetine-resistant "up" conformation (Soussia et al., 2018). Upon ML67 application, TREK1 does not become resistant to fluoxetine (Figures 7A,C). But TRAAK becomes sensitive to fluoxetine once stimulated by ML67 (Figures 7B,C). Similar results were obtained by swapping pCt between TREK1 and TRAAK. Upon application of ML67, TREK1pCt $t_{\text {TRAAK }}$ switches from resistant to sensitive to fluoxetine ( $\mathrm{I}_{\text {fluoxetine }} / \mathrm{I}_{\text {control }}=0.38 \pm$ 0.08 , Figures 7D,F). This action of ML67 is similar to the action of AA on TREK1pCt $t_{\text {TRAAK }}$ (Soussia et al., 2018). In contrast, TRAAKpCt $\mathrm{TREK}_{\mathrm{TR}}$ behaves like TREK1 and shows no changes in fluoxetine sensitivity upon ML67 application $\left(\mathrm{I}_{\text {fluoxetine }} / \mathrm{I}_{\text {control }}=0.56 \pm 0.05\right.$, Figures $\left.7 \mathbf{E}, \mathbf{F}\right)$. Taken together, these results show that ML67 is able to promote a shift of TRAAK from a fluoxetine-resistant state ("up") to a fluoxetine-sensitive state ("down") but is not able to change the conformational state of TREK1. Both AA and ML67 activate these channels in a pCtdependent manner but they do not have the same effect on the conformation of these channels, suggesting that two different allosteric mechanisms occur. One possibility is that the binding of ML67 to these channels stabilizes them in the "down" conformation. This is supported by the observation that the effect of fluoxetine on TREK1 is strongly enhanced in presence of ML67 (Figure 7A) suggesting a stabilization of the channel in the "down" state. It was recently shown that the inhibitory effect of norfluoxetine on TREK2 is neutralized by ML335 and not affected by 2-APB (Proks et al., 2021). Because we have not tested the effect of these openers on TREK1 sensitivity to

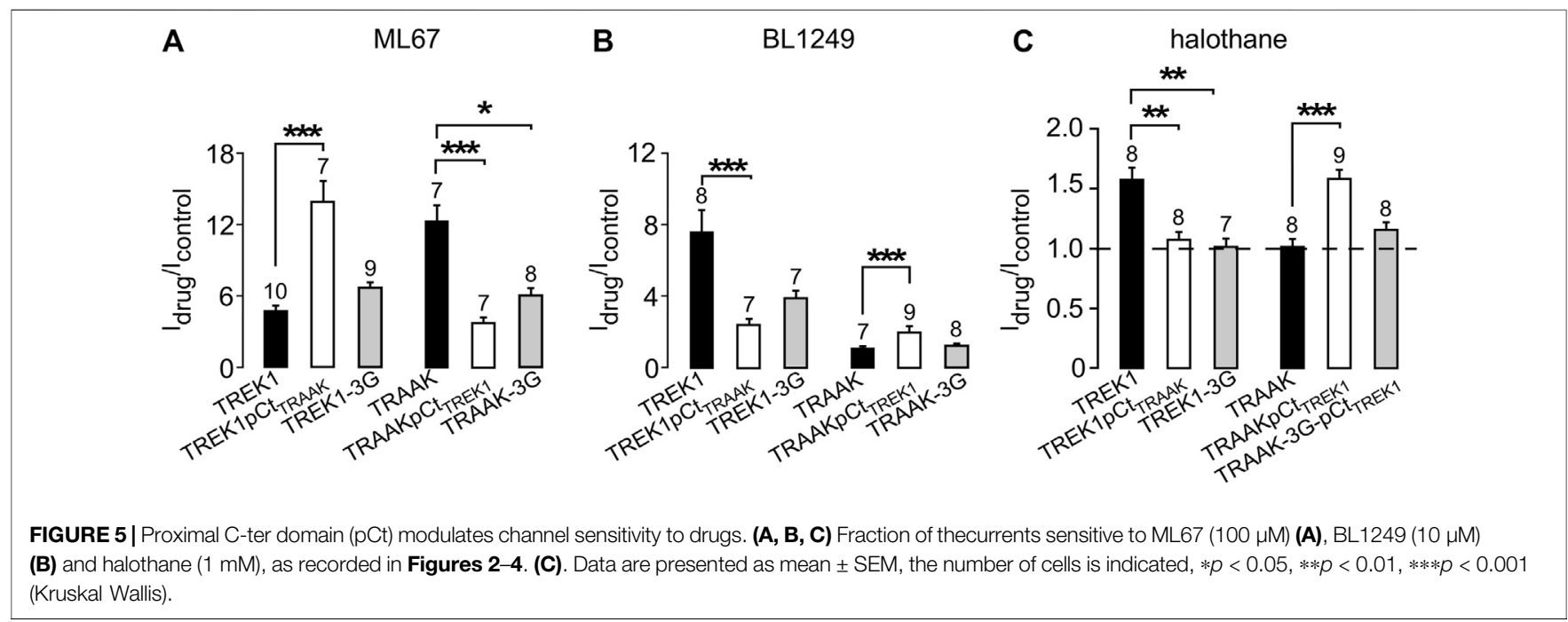



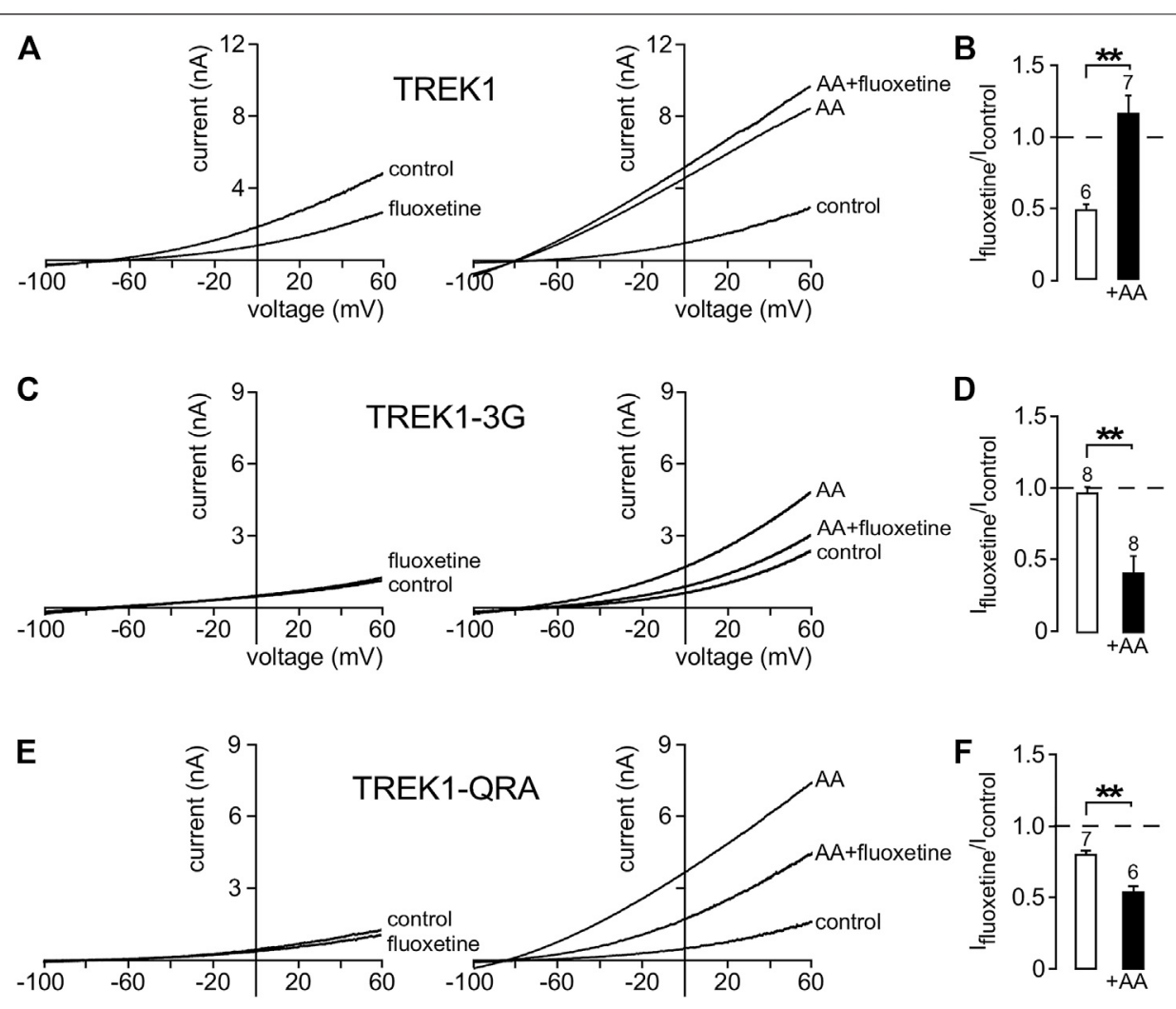

FIGURE 6 | State-dependent inhibitory effect of fluoxetine. (A, C, E) Representative whole-cell currents from cells transfected with TREK1 (A), TREK1-3G (C) and TREK1-QRA (E) channels in the presence or absence of $10 \mu \mathrm{M}$ fluoxetine on basal currents (left panel) and on currents stimulated by $A A(10 \mu M)$ (right panel). (B, D, F) Fraction of fluoxetine-sensitive current in the absence or presence of $\mathrm{AA}(10 \mu \mathrm{M})$. Data are presented as mean $\pm \mathrm{SEM}$, the number of cells is indicated, $* * p<0.01$ (MannWhitney test).
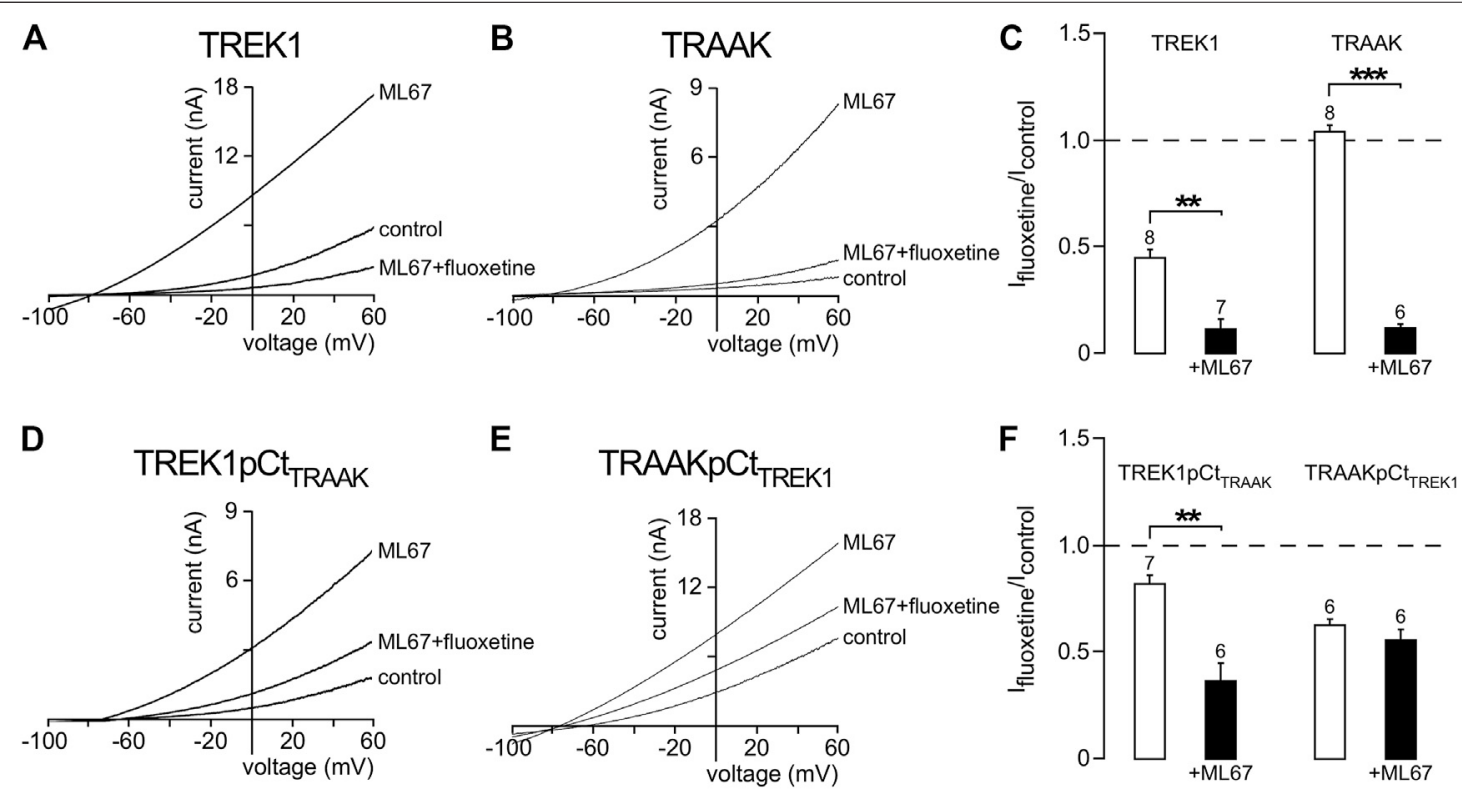

FIGURE 7 | State-dependent inhibitory effect of fluoxetine modulated by ML67. (A, B, D, E) Representative patch-clamp recordings from HEK cells expressing wild-type or mutated channels stimulated by ML67 $(100 \mu \mathrm{M})$ and in the presence of fluoxetine $(10 \mu \mathrm{M})$. (C, F) Fraction of fluoxetine-sensitive current in the absence or presence of ML67 $(100 \mu \mathrm{M})$. Data are presented as mean \pm SEM. $* * p<0.01, * * * p<0.001$, the number of cells is indicated (Mann-Whitney test). 

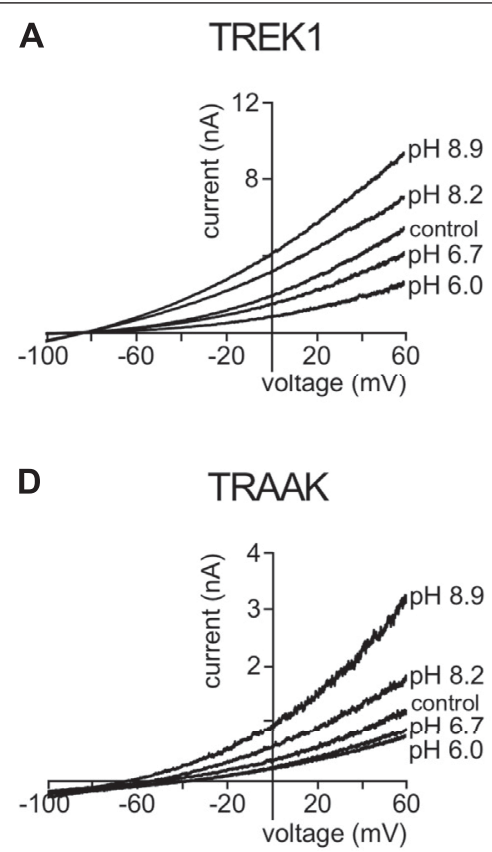

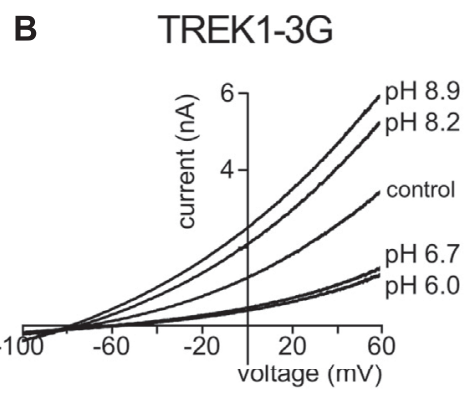

E

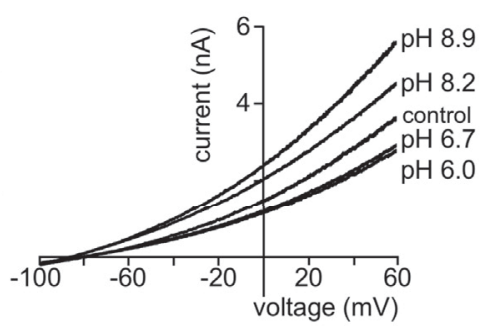

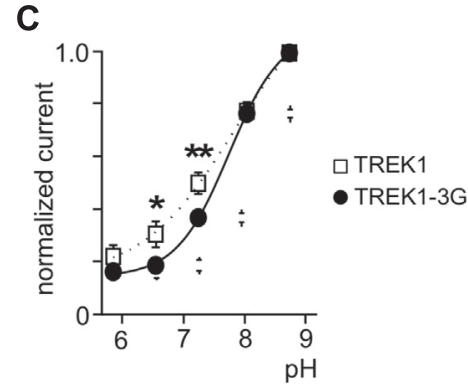

$\mathbf{F}$

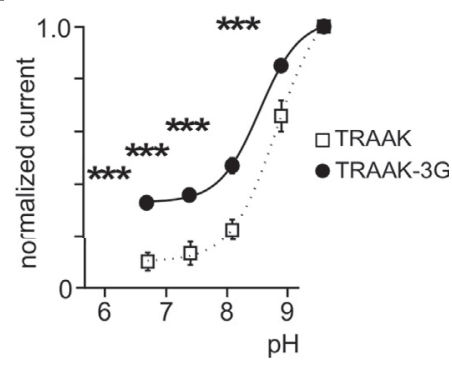

FIGURE 8 | Effect of extracellular pH. (A, B, D, E), Representative patch-clamp recordings from HEK cells expressing wild-type or mutated channels. Voltage ramps were applied from -100 to $60 \mathrm{mV}$ from a holding potential of $-80 \mathrm{mV}$. (C, F), Normalized pH response (at $0 \mathrm{mV}$ ) for indicated channels. Data are presented as mean \pm SEM. $* p<0.05, * * p<0.01, * * * p<0.001$ (Two-way ANOVA).

fluoxetine, we do not know how their binding affects the conformation of this channel.

\section{pCt Affects the Sensitivity of TREK1 and TRAAK to Extracellular pH}

TREK1 is activated by intracellular acidification through the E306 residue located in the pCt. The mutant TREK1E306A is resistant to intracellular acidification and less active than TREK1 (Honore et al., 2002). TREK1 is inhibited by extracellular acidification and stimulated by extracellular alkalinisation (Sandoz et al., 2009) (Figures 8A,C). Residues involved in this sensitivity are located on the extracellular side of the channel, close to the outer mouth of the pore. Here, we tested the role of pCt on the sensitivity of TREK1 and TRAAK to external $\mathrm{pH}$. We took advantage of the uncoupling of pCt and M4 in TREK1-3G to test a possible allosteric effect of pCt on the regulation by acidification. Inhibition by extracellular acidification was still observed in TREK1-3G in good agreement with the result reported by Bagriantsev et al. (2012), but the normalized remaining current at $\mathrm{pH} 7.4$ and 6.7 was a bit lower than TREK1 $(0.19 \pm 0.02$ vs $0.31 \pm 0.05$ at $\mathrm{pH} 6.7 ; 0.37 \pm 0.02$ vs $0.50 \pm 0.04$ at $\mathrm{pH} 7.4$, Figures $\mathbf{8 B}, \mathbf{C})$. Similar results were obtained by neutralizing K315 and E306 within the pCt of TREK1 (Woo et al., 2018). The $3 \mathrm{G}$ mutation also altered the sensitivity of TRAAK to extracellular $\mathrm{pH}$ (Figures 8D-F). The effect is stronger than on TREK1 with a significant fraction of TRAAK-3G being resistant to acidification (Figure 8F). This shows that extracellular $\mathrm{pH}$ and $\mathrm{pCt}$ trigger structural rearrangements that converge on a same and unique SF gate. However, the allosteric effects are less pronounced than between openers and pCt, suggesting multiple conformational states not equally responsive to extracellular and intracellular stimuli.

\section{DISCUSSION}

Many studies have focused on the structural mechanisms controlling the activity and pharmacology of TREK1, TREK2 and TRAAK channels. According to these studies, TREK/TRAAK channels have two main conformations, "up" and "down" (Brohawn et al., 2014; Lolicato et al., 2014) involving a movement of M4 directly coupled to the SF gate (Miller and Long, 2012; Dong et al., 2015; Zhuo et al., 2016). Our previous work has shown that pCt plays a critical role in channel conformation (Soussia et al., 2018). In basal conditions, TRAAK is in the fluoxetine-resistant "up" conformation whereas TREK1 is in the fluoxetine-sensitive "down" conformation (Soussia et al., 2018). In the same conditions, swapping pCt between these two channels leads TREK1pCtTRAAK to adopt the "up" conformation and TRAAKpCtTREK1 the "down" conformation. Here, we show that pCt also affects the sensitivity of TREK1 and TRAAK to extracellular $\mathrm{pH}$ and openers by acting on the SF gate. The recent co-crystallisation of TREK2 with a brominated BL1249 has shown that this drug binds to the "fenestration site" (Schewe et al., 2019). This site is located below the $\mathrm{P} 2$ pore helix and exposed by the "down" movement of M4 (Brohawn et al., 2012; Miller and Long, 2012; Brohawn et al., 2014; Lolicato et al., 2014). Like BL1249, ML67 also binds to the "fenestration site" (Bagriantsev et al., 2013; Schewe et al., 2019). Since this "fenestration site" is only accessible in the "down" state, we expected TREK1 to be sensitive to BL1249 and ML67, but not TRAAK. Surprisingly, TRAAK is sensitive to both 
openers like TREK1. However, TRAAK is more sensitive to ML67 than BL1249. Given that pCt affects channel conformation (Soussia et al., 2018), we wondered whether pCt could also affect the sensitivity of TREK1 and TRAAK channels to openers. Replacing TREK1 pCt by that of TRAAK increased the sensitivity of TREK1pCt $t_{\text {TRAAK }}$ to ML67 and decreased that to BL1249. In

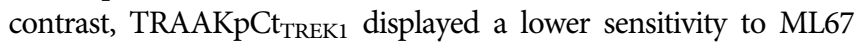
and a higher sensitivity to BL1249. Similar effects were observed with the 3G-mutation, which decouples pCt from the SF. These data indicate that pCt affects allosterically binding of these openers to the "fenestration site".

A recent study on volatile anaesthetics, which activate TREK1 (Patel et al., 1999) but not TRAAK, identified a binding site with a glycine as a key element at the M2-M3-M4 interface (Wague et al., 2020). Interestingly, this binding site corresponds to the "fenestration site", showing that different types of openers bind to this site. Swapping pCt between TREK1 and TRAAK or disrupting $\mathrm{pCt} / \mathrm{SF}$ coupling altered the sensitivity to halothane. These findings confirm the allosteric effects of pCt on SF gate via the "fenestration site". However, key questions remain unresolved: how does pCt affect drug binding to the "fenestration site"? And how does the binding of molecules to this "fenestration site" affect the SF state? A recent structural study on the TREK2/BL1249 complex gave new insights. The negatively charged tetrazole moiety of BL1249 promotes the SF conductive state by changing the coordination environment of $\mathrm{K}^{+}$ at position S1 and S4, increasing ion permeation (Schewe et al., 2019). ML67 contains a negatively charged moiety like BL1249 (Bagriantsev et al., 2013; Schewe et al., 2019), suggesting that BL1249 and ML67, and possibly halothane, may stabilize the SF gate in a conductive state through a similar mechanism. External $\mathrm{pH}$, which regulates TREK1, may also affect the SF gate by modulating ion permeation. Comparing the structures of the TREK-related TASK2 channel at $\mathrm{pH} 6.5$ and $\mathrm{pH} 8.5$ revealed that external acidification induces a distortion of the SF at the S0 and $\mathrm{S} 1$ positions that prevents $\mathrm{K}^{+}$to occupy these positions (Li et al., 2020). TREK 1 and TASK 2 are similarly sensitive to external $\mathrm{pH}$, suggesting that pCt in TREK1 may also affect the sensitivity to external $\mathrm{pH}$ in an allosteric manner. Consistent with this, we found that disrupting pCt/SF coupling with the 3G-mutation changed $\mathrm{pH}$-sensitivity of TREK1 and TRAAK. Not only openers bind to this "fenestration site" but also inhibitors, including fluoxetine and norfluoxetine (Miller and Long, 2012; Dong et al., 2015; Zhuo et al., 2016), indicating that the "fenestration site" is a dual-action site for its ligands. Identifying the precise molecular mechanism by which ligands influence the conformation site of the SF gate is key to the development of

\section{REFERENCES}

Bagriantsev, S. N., Ang, K. H., Gallardo-Godoy, A., Clark, K. A., Arkin, M. R., Renslo, A. R., et al. (2013). A High-Throughput Functional Screen Identifies Small Molecule Regulators of Temperature- and MechanoSensitive K2P Channels. ACS Chem. Biol. 8, 1841-1851. doi:10.1021/ $\mathrm{cb} 400289 \mathrm{x}$ novel modulators of TREK/TRAAK channels. It has to be noted that the "fenestration site" is composed of elements from both subunits of the active dimers (Natale et al., 2021), suggesting that homo and heterodimers could be specifically modulated (Blin et al., 2016; Lengyel et al., 2016; Levitz et al., 2016), enhancing the interest in developing drugs specifically targeting this region.

In summary, the allosteric effect of pCt on the stimulatory action of ML67, BL1249, AA and halothane shows that these stimuli converge to the same SF gate. This gate is the same as the gate controlled by extracellular $\mathrm{pH}$. Although the openers act through the same SF gate, they do not affect the conformational states of these channels in the same way. Previously, we showed that AA promotes the shift from the "up" to the "down" conformation of TREK1 and from the "down" to the "up" conformation of TRAAK (Soussia et al., 2018). Here, we showed that the 3G-mutation, which uncouples pCt from SF, changed the conformational state of TREK1 as expected. Surprisingly, and in contrast to AA, ML67 only promotes the transition from the "up" to the "down" conformation of TRAAK under basal conditions. TREK1 remains in the "down" conformation upon ML67 application. Taken collectively, these results provide further evidence that the crystallography identified "up" and "down" conformations do not correspond to open or closed states of these channels.

\section{DATA AVAILABILITY STATEMENT}

The raw data supporting the conclusions of this article will be made available by the authors, without undue reservation.

\section{AUTHOR CONTRIBUTIONS}

FL conceived the experiments and wrote the manuscript. FC, IS, DB, CF, SF, and DK contributed to the experimental work, conception of the experiments, and wrote the manuscript.

\section{FUNDING}

This work was funded by the Agence Nationale de la Recherche (Laboratory of Excellence "Ion Channel Science and Therapeutics", grant ANR-11-LABX-0015-01) and by the Fondation pour la Recherche Médicale (équipe labelisée FRM, grant EQ (U202003010587)).

Bagriantsev, S. N., Clark, K. A., and Minor, D. L., Jr. (2012). Metabolic and thermal Stimuli Control K(2P)2.1 (TREK-1) through Modular Sensory and Gating Domains. EMBO J. 31, 3297-3308. doi:10.1038/emboj.2012.171

Bagriantsev, S. N., Peyronnet, R., Clark, K. A., Honoré, E., and Minor, D. L., Jr. (2011). Multiple Modalities Converge on a Common Gate to Control K2P Channel Function. EMBO J. 30, 3594-3606. doi:10.1038/emboj.2011.230

Blin, S., Ben Soussia, I., Kim, E. J., Brau, F., Kang, D., Lesage, F., et al. (2016). Mixing and Matching TREK/TRAAK Subunits Generate Heterodimeric K2P Channels 
with Unique Properties. Proc. Natl. Acad. Sci. U S A. 113, 4200-4205. doi:10.1073/pnas.1522748113

Brohawn, S. G., Campbell, E. B., and MacKinnon, R. (2014). Physical Mechanism for Gating and Mechanosensitivity of the Human TRAAK K+ Channel. Nature 516, 126-130. doi:10.1038/nature14013

Brohawn, S. G., del Mármol, J., and MacKinnon, R. (2012). Crystal Structure of the Human K2P TRAAK, a Lipid- and Mechano-Sensitive K+ Ion Channel. Science 335, 436-441. doi:10.1126/science. 1213808

Dong, Y. Y., Pike, A. C., Mackenzie, A., McClenaghan, C., Aryal, P., Dong, L., et al. (2015). K2P Channel Gating Mechanisms Revealed by Structures of TREK-2 and a Complex with Prozac. Science 347, 1256-1259. doi:10.1126/ science. 1261512

Enyedi, P., and Czirják, G. (2010). Molecular Background of Leak K+ Currents: Two-Pore Domain Potassium Channels. Physiol. Rev. 90, 559-605. doi:10.1152/ physrev.00029.2009

Feliciangeli, S., Chatelain, F. C., Bichet, D., and Lesage, F. (2015). The Family of K2P Channels: Salient Structural and Functional Properties. J. Physiol. 593, 2587-2603. doi:10.1113/jphysiol.2014.287268

Heurteaux, C., Guy, N., Laigle, C., Blondeau, N., Duprat, F., Mazzuca, M., et al. (2004). TREK-1, a K+ Channel Involved in Neuroprotection and General Anesthesia. EMBO J. 23, 2684-2695. doi:10.1038/sj.emboj.7600234

Heurteaux, C., Lucas, G., Guy, N., El Yacoubi, M., Thümmler, S., Peng, X. D., et al. (2006). Deletion of the Background Potassium Channel TREK-1 Results in a Depression-Resistant Phenotype. Nat. Neurosci. 9, 1134-1141. doi:10.1038/ nn1749

Honoré, E., Maingret, F., Lazdunski, M., and Patel, A. J. (2002). An Intracellular Proton Sensor Commands Lipid- and Mechano-Gating of the $\mathrm{K}(+)$ Channel TREK-1. EMBO J. 21, 2968-2976. doi:10.1093/emboj/cdf288

Lengyel, M., Czirják, G., and Enyedi, P. (2016). Formation of Functional Heterodimers by TREK-1 and TREK-2 Two-Pore Domain Potassium Channel Subunits. J. Biol. Chem. 291, 13649-13661. doi:10.1074/ jbc.M116.719039

Levitz, J., Royal, P., Comoglio, Y., Wdziekonski, B., Schaub, S., Clemens, D. M., et al. (2016). Heterodimerization within the TREK Channel Subfamily Produces a Diverse Family of Highly Regulated Potassium Channels. Proc. Natl. Acad. Sci. U S A. 113, 4194-4199. doi:10.1073/pnas.1522459113

Li, B., Rietmeijer, R. A., and Brohawn, S. G. (2020). Structural Basis for pH Gating of the Two-Pore Domain K+ Channel TASK2. Nature 586, 457-462. doi:10.1038/s41586-020-2770-2

Lolicato, M., Riegelhaupt, P. M., Arrigoni, C., Clark, K. A., and Minor, D. L., Jr. (2014). Transmembrane helix Straightening and Buckling Underlies Activation of Mechanosensitive and Thermosensitive K(2P) Channels. Neuron 84, 1198-1212. doi:10.1016/j.neuron.2014.11.017

Maingret, F., Lauritzen, I., Patel, A. J., Heurteaux, C., Reyes, R., Lesage, F., et al. (2000a). TREK-1 Is a Heat-Activated Background K(+) Channel. EMBO J. 19, 2483-2491. doi:10.1093/emboj/19.11.2483

Maingret, F., Patel, A. J., Lesage, F., Lazdunski, M., and Honoré, E. (2000b). Lysophospholipids Open the Two-Pore Domain Mechano-Gated $\mathrm{K}(+)$ Channels TREK-1 and TRAAK. J. Biol. Chem. 275, 10128-10133. doi:10.1074/jbc.275.14.10128

Maingret, F., Patel, A. J., Lesage, F., Lazdunski, M., and Honoré, E. (1999). Mechano- or Acid Stimulation, Two Interactive Modes of Activation of the TREK-1 Potassium Channel. J. Biol. Chem. 274, 26691-26696. doi:10.1074/ jbc.274.38.26691

Miller, A. N., and Long, S. B. (2012). Crystal Structure of the Human Two-Pore Domain Potassium Channel K2P1. Science 335, 432-436. doi:10.1126/ science. 1213274

Natale, A. M., Deal, P. E., and Minor, D. L., Jr. (2021). Structural Insights into the Mechanisms and Pharmacology of K2P Potassium Channels. J. Mol. Biol. 433, 166995. doi:10.1016/j.jmb.2021.166995

Noël, J., Sandoz, G., and Lesage, F. (2011). Molecular Regulations Governing TREK and TRAAK Channel Functions. Channels (Austin) 5, 402-409. doi:10.4161/ chan.5.5.16469

Patel, A. J., Honoré, E., Lesage, F., Fink, M., Romey, G., and Lazdunski, M. (1999). Inhalational Anesthetics Activate Two-Pore-Domain Background K+ Channels. Nat. Neurosci. 2, 422-426. doi:10.1038/8084
Piechotta, P. L., Rapedius, M., Stansfeld, P. J., Bollepalli, M. K., Ehrlich, G., Erhlich G., et al. (2011). The Pore Structure and Gating Mechanism of K2P Channels. EMBO J. 30, 3607-3619. doi:10.1038/emboj.2011.268

Pope, L., Arrigoni, C., Lou, H., Bryant, C., Gallardo-Godoy, A., Renslo, A. R., et al. (2018). Protein and Chemical Determinants of BL-1249 Action and Selectivity for K2P Channels. ACS Chem. Neurosci. 9, 3153-3165. doi:10.1021/ acschemneuro.8b00337

Proks, P., Schewe, M., Conrad, L. J., Rao, S., Rathje, K., Rödström, K. E. J., et al. (2021). Norfluoxetine Inhibits TREK-2 K2P Channels by Multiple Mechanisms Including State-independent Effects on the Selectivity Filter Gate. J. Gen. Physiol. 153 (8), e202012812. doi:10.1085/jgp.202012812

Sandoz, G., Douguet, D., Chatelain, F., Lazdunski, M., and Lesage, F. (2009). Extracellular Acidification Exerts Opposite Actions on TREK1 and TREK2 Potassium Channels via a Single Conserved Histidine Residue. Proc. Natl. Acad. Sci. U S A. 106, 14628-14633. doi:10.1073/pnas.0906267106

Sandoz, G., Thümmler, S., Duprat, F., Feliciangeli, S., Vinh, J., Escoubas, P., et al. (2006). AKAP150, a Switch to Convert Mechano-, pH- and Arachidonic AcidSensitive TREK $\mathrm{K}(+)$ Channels into Open Leak Channels. EMBO J. 25, 5864-5872. doi:10.1038/sj.emboj.7601437

Schewe, M., Sun, H., Mert, Ü., Mackenzie, A., Pike, A. C. W., Schulz, F., et al. (2019). A Pharmacological Master Key Mechanism that Unlocks the Selectivity Filter Gate in K+ Channels. Science 363, 875-880. doi:10.1126/science.aav0569

Soussia, I. B., Choveau, F. S., Blin, S., Kim, E. J., Feliciangeli, S., Chatelain, F. C., et al. (2018). Antagonistic Effect of a Cytoplasmic Domain on the Basal Activity of Polymodal Potassium Channels. Front. Mol. Neurosci. 11, 301. doi:10.3389/ fnmol.2018.00301

Tertyshnikova, S., Knox, R. J., Plym, M. J., Thalody, G., Griffin, C., Neelands, T., et al. (2005). BL-1249 [(5,6,7,8-Tetrahydro-Naphthalen-1-Yl)-[2-(1h-Tetrazol5-Yl)-Phenyl]-Amine]: a Putative Potassium Channel Opener with BladderRelaxant Properties. J. Pharmacol. Exp. Ther. 313, 250-259. doi:10.1124/ jpet.104.078592

Wague, A., Joseph, T. T., Woll, K. A., Bu, W., Vaidya, K. A., Bhanu, N. V., et al. (2020). Mechanistic Insights into Volatile Anesthetic Modulation of K2P Channels. Elife 9. doi:10.7554/eLife.59839

Wang, W., Kiyoshi, C. M., Du, Y., Taylor, A. T., Sheehan, E. R., Wu, X., et al. (2020). TREK-1 Null Impairs Neuronal Excitability, Synaptic Plasticity, and Cognitive Function. Mol. Neurobiol. 57, 1332-1346. doi:10.1007/ s12035-019-01828-x

Woo, J., Jun, Y. K., Zhang, Y. H., Nam, J. H., Shin, D. H., and Kim, S. J. (2018). Identification of Critical Amino Acids in the Proximal C-Terminal of TREK-2 K+ Channel for Activation by Acidic pHi and ATP-dependent Inhibition. Pflugers Arch. 470, 327-337. doi:10.1007/ s00424-017-2072-1

Zhuo, R. G., Peng, P., Liu, X. Y., Yan, H. T., Xu, J. P., Zheng, J. Q., et al. (2016). Allosteric Coupling between Proximal C-Terminus and Selectivity Filter Is Facilitated by the Movement of Transmembrane Segment 4 in TREK-2 Channel. Sci. Rep. 6, 21248. doi:10.1038/srep21248

Conflict of Interest: The authors declare that the research was conducted in the absence of any commercial or financial relationships that could be construed as a potential conflict of interest.

Publisher's Note: All claims expressed in this article are solely those of the authors and do not necessarily represent those of their affiliated organizations, or those of the publisher, the editors and the reviewers. Any product that may be evaluated in this article, or claim that may be made by its manufacturer, is not guaranteed or endorsed by the publisher.

Copyright $\odot 2021$ Choveau, Ben Soussia, Bichet, Franck, Feliciangeli and Lesage. This is an open-access article distributed under the terms of the Creative Commons Attribution License (CC BY). The use, distribution or reproduction in other forums is permitted, provided the original author(s) and the copyright owner(s) are credited and that the original publication in this journal is cited, in accordance with accepted academic practice. No use, distribution or reproduction is permitted which does not comply with these terms. 